\title{
A COMPATIBILITY CONDITION BETWEEN INVARIANT RIEMANNIAN METRICS AND LEVI-WHITEHEAD DECOMPOSITIONS ON A COSET SPACE
}

\author{
BY \\ JOSEPH A. WOLF( ${ }^{(1)}$
}

0. Introduction. Let $M=G / K$ be an effective coset space of a connected Lie group by a compact subgroup. Then there may be many $G$-invariant riemannian metrics on $M$. But one expects the algebraic structure of the pair $(G, K)$ to have a strong influence on the curvatures of $M$ relative to any $G$-invariant riemannian metric. For example

(1) if $G$ is semisimple with finite center and $K$ is a maximal compact subgroup, then it is classical from symmetric space theory that all $G$-invariant riemannian metrics on $M$ have every sectional curvature $\leqq 0$;

(2) if $G$ is commutative then every $G$-invariant riemannian metric on $M$ is flat; and

(3) if $G$ is noncommutative and nilpotent then [7] every $G$-invariant riemannian metric on $M$ has sectional curvatures of both signs.

Those results are proved by choosing an $\operatorname{ad}_{G}(K)$-stable complement $\mathfrak{M}$ to the Lie algebra $\Re$ of $K$ inside the Lie algebra (S) of $G$, and by performing calculations in $\mathfrak{M}$ and in $\mathbb{S}$ in a manner justified by embedding $G$ in the orthonormal frame bundle of $M$. But at certain crucial parts of those calculations one must have (5) either semisimple or nilpotent. The idea in this paper is to create a setup in which the calculations can still be carried out, by requiring that the complement $\mathfrak{M}$ split as

where

$$
\mathfrak{M}=(\mathfrak{M} \cap \mathfrak{R})+(\mathfrak{M} \cap \mathfrak{L}) \text { orthogonal direct sum, }
$$

and

$$
\text { (S) }=\mathfrak{R}+\mathfrak{Q} \text { is a Levi-Whitehead decomposition }
$$

$\mathfrak{M} \cap \Re$ contains the nilpotent radical of $\mathbb{S}$.

$\$ 2$ is a study of the circumstances under which $\mathfrak{M}$ can be chosen, and the LeviWhitehead decomposition $\mathscr{B}=\mathfrak{R}+\mathfrak{Q}$ can be chosen, so that $M$ has such an orthogonal splitting. We describe those circumstances by the condition (2.2) that the invariant riemannian metric on $M$ be "consistent" with $\mathscr{S}=\mathfrak{R}+\mathfrak{L}$.

Given the consistency condition (2.2), our main result (Theorem 3.9) says that every unit vector $X \in \mathfrak{M} \cap \mathfrak{R}$, orthogonal to the nilpotent radical $\mathfrak{N}$ of $\mathscr{G}$, is a

Received by the editors May 21, 1968.

${ }^{(1)}$ Research supported by an Alfred P. Sloan Research Fellowship and by National Science Foundation Grant GP-8008. 
direction of negative mean curvature on $M$. Our applications ( $\$ 4)$ essentially consist of observing that, if $M$ has mean curvature $\geqq 0$ everywhere, then the consistency condition implies $\mathfrak{N}=(\mathfrak{M} \cap \mathfrak{R})$, i.e. $\mathfrak{R}=\mathfrak{R}+(\mathfrak{R} \cap \mathfrak{R})$ semidirect sum. The most striking of the applications is Theorem 4.4, which says:

Let $M$ be a connected Riemannian manifold that has a solvable transitive group of isometries. Then the following conditions are equivalent.

(i) $M$ has mean curvature $\geqq 0$ everywhere.

(ii) $M$ has every sectional curvature $\geqq 0$.

(iii) $M$ has every sectional curvature zero.

(iv) $M$ is isometric to the product of an euclidean space and a flat riemannian torus.

Theorem 4.7 adds negative curvature conditions in case $M$ has a transitive nilpotent group of isometries, extending the results of [7] to mean curvature.

1. Definitions and notation. IS is a real Lie algebra. We have the nilpotent radical $\mathfrak{R}$ and the solvable radical $\mathfrak{R}$, characteristic nilpotent and solvable ideals in $\mathfrak{S}$ defined by

$\mathfrak{N}$ is the union of the nilpotent ideals of $\mathscr{B S}$,

$\mathfrak{R}$ is the union of the solvable ideals of $\mathbb{B S}$.

The basic facts on $\mathfrak{R}$ and $\Re$ are the following.

(1.1) If C is a fully reducible group of automorphisms of (s), then there are C-invariant semisimple subalgebras $\mathfrak{L} \subset \mathbb{B S}$ that map isomorphically onto $\mathbb{S} / \mathfrak{R}$ under the projection $\varphi: \mathbb{S} \rightarrow \mathbb{S} / \Re$, and any two such subalgebras are conjugate by an automorphism $\operatorname{ad} \leftrightarrow(\exp n)$ of $(S)$ where $n \in \mathfrak{N}$ is left fixed by every $c \in C$.

The existence is due to G. D. Mostow [3, Corollary 5.2], and the conjugacy statement is the result [5, Theorem 4] of E. J. Taft. In general a semisimple subalgebra $\mathfrak{L} \subset \mathbb{S}$ such that $\varphi: \mathfrak{L} \cong \mathbb{S} / \mathfrak{R}$ is called a Levi factor of $\mathfrak{S}$, and the Levi factors of $(S)$ are just the maximal semisimple subalgebras.

(1.2) If $\mathfrak{Q}$ is a Levi factor of $\mathfrak{G}$, then $\mathbb{S}=\mathfrak{R}+\mathfrak{Q}$ semidirect sum, $\mathfrak{R}+\mathfrak{L}$ (semidirect) is an ideal in $\mathfrak{S}$, and the derived algebra $[\mathfrak{S}, \mathbb{S}] \subset \mathfrak{N}+\mathfrak{L}$.

The first assertion is immediate and the second follows from $\mathfrak{R} \subset \mathfrak{R}$. For the third, one notes that $[\Re, \Re] \subset \mathfrak{N}$ by Ado's Theorem and that $\operatorname{ad}_{\mathfrak{S}}(\mathfrak{L})$ normalizes $\operatorname{ad}_{\Re}(\Re)$ in the derivation algebra of $\Re$.

Let $M=G / K$ be a coset space of a Lie group by a closed subgroup. $\mathfrak{A} \subset \mathbb{B}$ are the Lie algebras of $K \subset G$. An $\operatorname{ad}_{G}(K)$-invariant subspace $\mathfrak{M} \subset \mathbb{S}$ such that $\mathbb{S}=\mathfrak{M}+\mathfrak{R}$ (vector space direct sum), is called an invariant complement for $K$. If an invariant complement for $K$ exists, then $M=G / K$ is called a reductive coset space.

$K$ is called a reductive subgroup of $G$ in case the $\operatorname{group} \operatorname{ad}_{G}(K)$ of linear transformations of $\mathscr{B S}$ is fully reducible. If $K$ is a reductive subgroup of $G$, then $\operatorname{ad}_{G}(K) \Re$ 
$=\mathfrak{\Omega}$ implies that $M=G / K$ is a reductive coset space. The converse fails in the example

$$
G=S L(2, R) \quad \text { and } \quad K=\left\{\left(\begin{array}{ll}
1 & n \\
0 & 1
\end{array}\right): n \in Z\right\}
$$

However, compact subgroups, and semisimple subgroups with only finitely many components, are reductive subgroups.

2. The compatibility condition. We can now define the compatibility conditions with which we will operate. $M=G / K$ is a coset space of a Lie group by a closed subgroup. $\mathfrak{L}$ is a Levi factor of $\mathfrak{S}$ and $\mathfrak{M}$ is an invariant complement for $K$. If

$$
\mathfrak{M}=(\mathfrak{M} \cap \mathfrak{R})+(\mathfrak{M} \cap \mathfrak{L}) \quad \text { and } \quad \mathfrak{R}=(\mathfrak{M} \cap \mathfrak{R})+(\mathfrak{R} \cap \mathfrak{R})
$$

then we say that $\mathfrak{L}$ splits $\mathfrak{M}$ and that $\mathfrak{M}$ is split by the Levi-Whitehead decomposition $\mathfrak{G}=\mathfrak{R}+\mathfrak{Q}$. Suppose further that we have a $G$-invariant pseudo-riemannian metric $d s^{2}$ on $M$. Represent $d s^{2}$ by an $\operatorname{ad}_{G}(K)$-invariant inner product $\langle$,$\rangle on \mathfrak{M}$. If

$$
\begin{gathered}
\mathfrak{M}=(\mathfrak{M} \cap \mathfrak{R})+(\mathfrak{M} \cap \mathfrak{L}), \\
\mathfrak{R}=(\mathfrak{M} \cap \mathfrak{R})+(\mathfrak{N} \cap \mathfrak{R}) \text { and }\langle\mathfrak{M} \cap \mathfrak{R}, \mathfrak{M} \cap \mathfrak{L}\rangle=0,
\end{gathered}
$$

then we say that $\mathfrak{L}$ splits $\mathfrak{M}$ orthogonally and that $d s^{2}$ is consistent with the LeviWhitehead decomposition $\mathbb{S}=\mathfrak{R}+\mathfrak{Q}$.

2.3. Proposition. Let $K$ be a closed reductive subgroup of a Lie group $G$. Then for every $\operatorname{ad}_{G}(K)$-invariant Levi factor $\mathfrak{L}$ of $\mathfrak{S}$, there exists an invariant complement $\mathfrak{M}$ for $K$, such that $\mathfrak{L}$ splits $\mathfrak{M}$. If $d s^{2}$ is a G-invariant pseudo-riemannian metric on $G / K, \varphi:(S) \rightarrow B S / \Re$ is the projection, and the representations of $K$ on $\Re /(\Re \cap \Re)$ and $\mathfrak{Q} /\left(\mathfrak{Q} \cap \varphi^{-1} \varphi \mathfrak{R}\right)$ are disjoint, then it is automatic that $\mathfrak{L}$ splits $\mathfrak{M}$ orthogonally and $d s^{2}$ is consistent with $\mathbb{H}=\mathfrak{R}+\mathfrak{Q}$.

Proof. Mostow's result (1.1) provides an $\operatorname{ad}_{G}(K)$-invariant Levi factor $\mathfrak{L}$ of $\mathfrak{S}$. As $K$ is reductive in $G$ we have $\operatorname{ad}_{G}(K)$-invariant direct sum decompositions

Now define

$$
\Re=\mathfrak{M}_{1}+(\mathfrak{R} \cap \mathfrak{R}) \quad \text { and } \quad \mathfrak{G} / \mathfrak{R}=\mathfrak{M}_{2}^{\prime}+\varphi(\mathfrak{R}) \text {. }
$$

$$
\mathfrak{M}_{2}=\mathfrak{L} \cap \varphi^{-1}\left(\mathfrak{M}_{2}^{\prime}\right) \quad \text { and } \quad \mathfrak{M}=\mathfrak{M}_{1}+\mathfrak{M}_{2}
$$

Then $\operatorname{ad}_{G}(K) \mathfrak{M}_{i}=\mathfrak{M}_{i}$ so $\mathfrak{M}$ is an $\operatorname{ad}_{G}(K)$-invariant subspace of $\mathscr{S}$ that satisfies (2.1). If $x \in \mathfrak{M} \cap \mathfrak{R}$ then $\varphi(x) \in \mathfrak{M}_{2}^{\prime} \cap \varphi(\Re)=0$ so $x \in \mathfrak{R}$; then $x \in \mathfrak{M}_{1} \cap(\Re \cap \Re)=0$ so $x=0$; thus $\mathfrak{M} \cap \mathfrak{R}=0$. On the other hand

$$
\operatorname{dim} \mathfrak{R}=\operatorname{dim} \mathfrak{M}_{1}+\operatorname{dim}(\Re \cap \Re)
$$

and

$$
\begin{aligned}
\operatorname{dim} \mathfrak{S} / \Re & =\operatorname{dim} \mathfrak{M}_{2}^{\prime}+\operatorname{dim} \varphi(\Re)=\operatorname{dim} \mathfrak{M}_{2}+\operatorname{dim} \varphi(\Re) \\
& =\operatorname{dim} \mathfrak{M}_{2}-\operatorname{dim}(\Re \cap \Re)+\operatorname{dim} \Re
\end{aligned}
$$

so

$$
\operatorname{dim} \mathfrak{S}=\operatorname{dim} \mathfrak{R}+\operatorname{dim} \mathfrak{S} / \mathfrak{R}=\operatorname{dim} \mathfrak{M}+\operatorname{dim} \mathfrak{R} .
$$


Thus $\mathfrak{M}$ is a vector space complement to $\mathfrak{R}$ in $(\mathfrak{S}$. Now $\mathfrak{M}$ is an invariant complement for $K$ such that $\mathfrak{L}$ splits $\mathfrak{M}$.

Let $d s^{2}$ and the inner product $\langle,>$ on $\mathfrak{M}$ be given. The representation of $K$ on $\mathfrak{R} /(\mathfrak{R} \cap \mathfrak{\Re})$ is the representation $\left.\operatorname{ad}_{G}\right|_{K}$ on $\mathfrak{M}_{1}$; the representation of $K$ on $\mathfrak{L} /\left(\mathfrak{L} \cap \varphi^{-1} \varphi \mathfrak{R}\right)$ is $\left.\operatorname{ad}_{G}\right|_{K}$ on $\mathfrak{M}_{2}$. If those two are disjoint then necessarily $\left\langle\mathfrak{M}_{1}, \mathfrak{M}_{2}\right\rangle=0$. Q.E.D.

We reformulate the metric portion of Proposition 2.3.

2.4. Proposition. Let $M=G / K$ be a homogeneous pseudo-riemannian manifold with metric $d s^{2}$, where $K$ is a reductive subgroup of $G$. Let $\mathcal{O}$ be the base point, $M_{\mathcal{O}}$ the tangent space at $\mathcal{O}, \chi$ the linear isotropy representation of $K$ on $M_{\mathcal{O}}$, and $R_{\mathcal{O}}$ the subspace of $M_{\mathcal{O}}$ spanned by vector fields from elements of the solvable radical of $(\dot{S}$. Suppose that the representations of $K$ induced by $\chi$, on $R_{\mathcal{O}}$ and on $M_{\mathcal{O}} / R_{\mathcal{O}}$, are disjoint. Then $M_{\mathcal{O}}=R_{\mathcal{O}}+R_{\mathcal{O}}^{\perp}$ and $d s^{2}$ is consistent with every Levi-Whitehead decomposition (S) $=\mathfrak{R}+\mathfrak{Q}$ for which $\operatorname{ad}_{G}(K) \cdot \mathfrak{L}=\mathfrak{Q}$.

For, in the notation of the proof of Proposition 2.3, $R_{\mathcal{O}}$ is spanned by the vector fields from $\mathfrak{M}_{1}$ while $R_{\mathscr{O}}^{\perp}$ is spanned by those from $\mathfrak{M}_{2}$.

In the riemannian case we will be able to arrange that $\mathfrak{M}_{1}=\mathfrak{M} \cap \mathfrak{R}$ contain the nilpotent radical $\mathfrak{R}$. For that, we need a technical lemma.

2.5. LeMMA. In a connected nilpotent Lie group every compact subgroup is central.

Proof. Let $N$ be the connected nilpotent Lie group, $\pi: \tilde{N} \rightarrow N$ the universal Lie group covering, and $\Gamma$ the kernel of $\pi$. Then $\Gamma$ is a discrete central subgroup of $\tilde{N}$. Let $C$ be a maximal compact subgroup of $N$ and $\widetilde{C}=\pi^{-1}(C)$. Then $C$ is a torus group, $\widetilde{C}$ is a simply connected commutative subgroup of $\tilde{N}$, and $\Gamma \subset \widetilde{C}$ such that $C=\tilde{C} / \Gamma$ compact. As $\Gamma$ is central in $\tilde{N}$, and as $\tilde{N}$ is nilpotent, now $\widetilde{C}$ is central in $\tilde{N}$, so $C$ is central in $N$. If $E$ is any compact subgroup of $N$ we have $n \in N$ such that $n E n^{-1} \subset C$, so $E \subset n^{-1} C n=C$, proving $E$ central in $N$. Q.E.D.

2.6. Proposition. Let $M=G / K$ be an effective coset space of a connected Lie group by a compact subgroup. Then, for any $\operatorname{ad}_{G}(K)$-invariant Levi factor $\mathfrak{Q}$ of (S), there is an invariant complement $\mathfrak{M}$ for $K$ such that

$$
\mathfrak{M}=(\mathfrak{M} \cap \mathfrak{R})+(\mathfrak{M} \cap \mathfrak{Q}), \quad \mathfrak{R}=(\mathfrak{M} \cap \mathfrak{R})+(\mathfrak{R} \cap \mathfrak{R}), \quad \text { and } \quad \mathfrak{R} \subset \mathfrak{M} \cap \mathfrak{R}
$$

In particular, if $G$ is a group of isometries for a riemannian metric on $M$, and if the metric is consistent with the Levi-Whitehead decomposition $\mathfrak{S}=\mathfrak{R}+\mathfrak{L}$, then in addition we can choose $\mathfrak{M}$ so that $\mathfrak{M} \cap \mathfrak{R}$ has a subspace $\mathfrak{A}$ such that

(2.8) $\mathfrak{M}=\mathfrak{R}+\mathfrak{A}+(\mathfrak{R} \cap \mathfrak{L}), \quad \mathfrak{M} \cap \mathfrak{R}=\mathfrak{N}+\mathfrak{A}, \quad$ orthogonal direct sums.

Proof. Following Proposition 2.3 we take $\mathfrak{M}=\mathfrak{M}_{1}+\mathfrak{M}_{2}$, invariant complement for $K$ split by $\mathfrak{L}$, where $\mathfrak{M}_{1}$ is any $\operatorname{ad}_{G}(K)$-invariant complement to $\Re \cap \mathfrak{R}$ in $\Re$. 
Let $N$ be the analytic subgroup of $G$ for $\Re$. Then $N$ is closed in $G$, so $N \cap K$ is compact. Let $T$ be a maximal compact subgroup of $N$. It contains $N \cap K$ and is central in $N$ by Lemma 2.5. Thus $T$ is unique, hence normal in $G$. As $T$ is a torus and $G$ is connected now $T$ is central in $G$. Thus $N \cap K$ is central in $G$. But $G$ acts effectively on $M$, so $K$ contains no nontrivial normal subgroup of $G$. That proves $N \cap K=\{1\}$. In particular $\mathfrak{R} \cap \mathfrak{R}=0$. Thus $\mathfrak{R}=\mathfrak{R}+\mathfrak{A}+(\mathfrak{R} \cap \mathfrak{\Re}) \operatorname{ad}_{G}(K)$-invariant direct sum, where $\mathfrak{A}$ is any invariant complement to $\mathfrak{R}+(\mathfrak{R} \cap \mathfrak{\Re})$. For $(2.7)$ we just choose $\mathfrak{M}_{1}=\mathfrak{R}+\mathfrak{A}$.

Suppose further that $d s^{2}$ is consistent with $\mathbb{G}=\mathfrak{R}+\mathfrak{L}$. Then we have another choice, say $\mathfrak{M}^{*}=\mathfrak{M}_{1}^{*}+\mathfrak{M}_{2}^{*}$, of invariant complement for $K$, with $\left\langle\mathfrak{M}_{1}^{*}, \mathfrak{M}_{2}^{*}\right\rangle=0$ and $\Re=\mathfrak{M}_{1}^{*}+(\Re \cap \Re)$. If $\psi: \mathfrak{M} \cong \mathscr{S} / \Re$ and $\psi^{*}: \mathfrak{M}^{*} \cong \mathfrak{S} / \mathfrak{\Re}$ are induced by the projection $\mathscr{G} \rightarrow \mathfrak{G} / \mathfrak{R}$, now $\psi^{-1} \psi^{*}: \mathfrak{M}^{*} \rightarrow \mathfrak{M}$ is a linear isometry carrying $\mathfrak{M}_{i}^{*}$ to $\mathfrak{M}_{i}$. Thus $\left\langle\mathfrak{M}_{1}, \mathfrak{M}_{2}\right\rangle=0$, and we obtain (2.8) by choosing $\mathfrak{A}$ to be the orthocomplement of $\mathfrak{N}$ in $\mathfrak{M}_{1}$. Q.E.D.

We will view the space $\mathfrak{A}$ of $(2.8)$ as the "gap" between nilpotent and solvable radicals of $\mathscr{S}$, taken modulo $\Re$.

3. Mean curvature along the gap between the nilpotent and solvable radicals. We compute the mean curvature of a homogeneous riemannian manifold along a direction in the solvable radical complementary to the nilpotent radical. This is done by specializing the following general calculation to the case where the riemannian metric is consistent with a Levi-Whitehead decomposition.

3.1. Lemma. Let $\left(M, d s^{2}\right)$ be a connected n-dimensional riemannian homogeneous space. Let $\mathcal{O} \in M$. Let $G$ be a connected transitive group of isometries of $M$ and let $M_{\mathscr{O}}^{\prime}$ denote the subspace of $M_{\mathcal{O}}$ consisting of tangent vectors $Y_{\mathcal{O}}$ where $Y$ is in the derived algebra [GS, (S)]. If $X_{\mathcal{O}} \in M_{\mathcal{O}}$ is a unit vector orthogonal to $M_{\mathcal{O}}^{\prime}$, then the mean curvature

$$
\begin{aligned}
(n-1) \boldsymbol{k}\left(X_{\mathcal{O}}\right)= & \sum_{i}\left\langle\left[\left\{\left[X, E_{i}\right]_{\mathfrak{R}}+\frac{1}{4}\left[X, E_{i}\right]_{\mathfrak{M}}\right\}, X\right]_{\mathfrak{M}}, E_{\mathfrak{i}}\right\rangle \\
& -\frac{1}{2} \sum_{i}\left\|\left[X, E_{i}\right]_{\mathfrak{M}}\right\|^{2} \\
& -\frac{1}{4} \sum_{i, j}\left\langle\left[X, E_{i}\right]_{\mathfrak{M}}, E_{j}\right\rangle \cdot\left\langle\left[X, E_{j}\right]_{\mathfrak{M}}, E_{\mathfrak{i}}\right\rangle,
\end{aligned}
$$

where $\mathfrak{M} \subset \mathbb{C S}$ is an invariant complement to $K, X \in \mathfrak{M}$ represents $X_{\mathcal{O}},\langle$,$\rangle is the$ inner product on $\mathfrak{M}$ from $d^{2}$, and $\left\{E_{i}\right\}$ is any orthonormal basis of $\mathfrak{M}$ containing $X$.

Proof. We follow the method of Nomizu [4], using the notation

$\alpha: \mathfrak{M} \times \mathfrak{M} \rightarrow \mathfrak{M}$ for the connection function,

$\mathscr{U}: \mathfrak{M} \times \mathfrak{M} \rightarrow \mathfrak{M}$ for the symmetric part of $\alpha$,

$\mathscr{R}: \mathfrak{M} \times \mathfrak{M} \times \mathfrak{M} \rightarrow \mathfrak{M}$ for the curvature tensor.

Now $(n-1) k(X)=\sum_{i \neq i_{0}} K_{i}=-\sum_{i \neq i_{0}}\left\langle\mathscr{R}\left(X, E_{i}\right) X, E_{i}\right\rangle=-\sum_{i}\left\langle\mathscr{R}\left(X, E_{i}\right) X, E_{i}\right\rangle$ where 
$\left\{E_{i}\right\}$ is an orthonormal basis of $\mathfrak{M}$, where $X=E_{i_{0}}$, and where $K_{i}$ is the sectional curvature of the tangent 2-plane spanned by $X$ and $E_{i}$. Thus

$$
(n-1) k(X)=-\sum_{i}\left\langle\mathscr{R}\left(X, E_{i}\right) X, E_{i}\right\rangle .
$$

Using [4, formulae 9.6 and 13.1] and correcting a misprint in the latter,

$$
\begin{aligned}
\mathscr{R}\left(X, E_{i}\right) X & =\alpha\left(X, \alpha\left(E_{i}, X\right)\right)-\alpha\left(E_{i}, \alpha(X, X)\right)-\alpha\left(\left[X, E_{i}\right]_{\mathfrak{R},}, X\right)-\left[\left[X, E_{i}\right]_{\mathfrak{R}}, X\right] . \\
\alpha(S, T) & =\frac{1}{2}[S, T]_{\mathfrak{R}}+\mathscr{U}(S, T) . \\
\mathscr{U}(S, T) & =-\frac{1}{2} \sum_{j}\left\{\left\langle\left[S, E_{j}\right]_{\mathfrak{M},}, T\right\rangle+\left\langle\left[T, E_{j}\right]_{\mathfrak{M}}, S\right\rangle\right\} E_{j} .
\end{aligned}
$$

Our hypothesis on $X$ and $X_{\mathcal{O}}$ is that $\left\langle X,[A, B]_{\mathfrak{A} R}\right\rangle=0$ for all $A, B \in \mathfrak{M}$. In particular

$$
\mathscr{U}(X, S)=\mathscr{U}(S, X)=-\frac{1}{2} \sum_{j}\left\langle\left[X, E_{j}\right]_{\mathfrak{R}}, S\right\rangle E_{j}
$$

Thus $\alpha(X, X)=0$. Substituting that into (3.3) we have

$$
\begin{aligned}
(n-1) \boldsymbol{k}(X)= & -\sum_{i}\left\langle\alpha\left(X, \alpha\left(E_{i}, X\right)\right), E_{i}\right\rangle \\
& +\sum_{i}\left\langle\alpha\left(\left[X, E_{i}\right]_{\mathfrak{M}}, X\right), E_{i}\right\rangle \\
& +\sum_{i}\left\langle\left[\left[X, E_{i}\right]_{\mathbb{R}}, X\right], E_{i}\right\rangle .
\end{aligned}
$$

In order to evaluate the right-hand side of (3.5) we define coefficients $b_{j k}$ by $\left[X, E_{j}\right]_{\mathfrak{R} R}=\sum_{k} b_{j k} E_{k}$. Then, using (3.4),

$$
\begin{aligned}
2 \sum_{i}\left\langle\left[X, \mathscr{U}\left(E_{i}, X\right)\right]_{\mathfrak{M}}, E_{i}\right\rangle & =-\sum_{i, j}\left\langle\left[X,\left\langle\left[X, E_{j}\right]_{\mathfrak{M}}, E_{i}\right\rangle E_{j}\right]_{\mathfrak{M}}, E_{i}\right\rangle \\
& =-\sum_{i, j}\left\langle\left[X, E_{j}\right]_{\mathfrak{M}}, E_{\mathfrak{i}}\right\rangle^{2}=-\sum_{i, j} b_{j i}^{2} \\
& =-\sum_{j}\left(\sum_{i} b_{j i}^{2}\right)=-\sum_{j}\left\|\left[X, E_{j}\right]_{\mathfrak{M}}\right\|^{2} \\
& =-\sum_{i}\left\|\left[X, E_{i}\right]_{\mathfrak{R}}\right\|^{2}=\sum_{i}\left\langle\left[X, E_{i}\right]_{\mathfrak{R}},\left[E_{i}, X\right]_{\mathfrak{R}}\right\rangle \\
& =+\sum_{i, j}\left\langle\left\{\left\langle\left[X, E_{j}\right]_{\mathfrak{R}},\left[E_{i}, X\right]_{\mathfrak{R}}\right\rangle\right\} E_{j}, E_{i}\right\rangle \\
& =-2 \sum_{i}\left\langle\mathscr{U}\left(X,\left[E_{i}, X\right]_{\mathfrak{R}}\right), E_{i}\right\rangle .
\end{aligned}
$$

In other words

$$
-\frac{1}{2} \sum_{i}\left\langle\left[X, \mathscr{U}\left(E_{i}, X\right)\right]_{\mathfrak{M}}, E_{i}\right\rangle-\frac{1}{2} \sum_{i}\left\langle\mathscr{U}\left(X,\left[E_{i}, X\right]_{\mathfrak{M}}\right), E_{i}\right\rangle=0 .
$$


Using (3.4) and (3.6) we compute

$$
\begin{aligned}
&-\sum_{i}\left\langle\alpha\left(X, \alpha\left(E_{\mathfrak{i}}, X\right)\right), E_{i}\right\rangle \\
&=-\frac{1}{4} \sum_{i}\left\langle\left[X,\left[E_{i}, X\right]_{\mathfrak{R}}\right]_{\mathfrak{M}}, E_{\mathfrak{i}}\right\rangle-\sum_{i}\left\langle\mathscr{U}\left(X, \mathscr{U}\left(E_{i}, X\right)\right), E_{\mathfrak{i}}\right\rangle \\
&-\frac{1}{2} \sum_{i}\left\langle\left[X, \mathscr{U}\left(E_{i}, X\right)\right]_{\mathfrak{M}}, E_{i}\right\rangle-\frac{1}{2} \sum_{i}\left\langle\mathscr{U}\left(X,\left[E_{i}, X\right]_{\mathfrak{M}}\right), E_{\mathfrak{i}}\right\rangle \\
&=-\frac{1}{4} \sum_{i}\left\langle\left[X,\left[E_{i}, X\right]_{\mathfrak{M}}\right]_{\mathfrak{M}}, E_{i}\right\rangle-\sum_{i}\left\langle\mathscr{U}\left(X, \mathscr{U}\left(E_{i}, X\right)\right), E_{\mathfrak{i}}\right\rangle \\
&=-\frac{1}{4} \sum_{i}\left\langle\left[X,\left[E_{i}, X\right]_{\mathfrak{R}}\right]_{\mathfrak{M}}, E_{i}\right\rangle-\frac{1}{4} \sum_{i, j}\left\langle\left[X, E_{\mathfrak{i}}\right]_{\mathfrak{M}}, E_{j}\right\rangle\left\langle\left[X, E_{j}\right]_{\mathfrak{M}}, E_{i}\right\rangle .
\end{aligned}
$$

That gives us the first summand of the right-hand side of (3.5):

$$
\begin{aligned}
-\sum_{i}\left\langle\alpha\left(X, \alpha\left(E_{i}, X\right)\right), E_{\mathfrak{i}}\right\rangle= & -\frac{1}{4} \sum_{i}\left\langle\left[\left[X, E_{\mathfrak{i}}\right]_{\mathfrak{M}}, X\right]_{\mathfrak{M},}, E_{\mathfrak{i}}\right\rangle \\
& -\frac{1}{4} \sum_{i, j}\left\langle\left[X, E_{\mathfrak{i}}\right]_{\mathfrak{M}}, E_{j}\right\rangle\left\langle\left[X, E_{j}\right]_{\mathfrak{M}}, E_{\mathfrak{i}}\right\rangle .
\end{aligned}
$$

The second summand of the right-hand side of (3.5) is, again using (3.4),

$$
\begin{aligned}
\sum_{i}\left\langle\alpha\left(\left[X, E_{i}\right]_{\mathfrak{M}}, X\right), E_{i}\right\rangle \\
\quad=\frac{1}{2} \sum_{i}\left\langle\left[\left[X, E_{i}\right]_{\mathfrak{M} i}, X\right]_{\mathfrak{M}}, E_{i}\right\rangle-\frac{1}{2} \sum_{i, j}\left\langle\left\{\left\langle\left[X, E_{j}\right]_{\mathfrak{M}},\left[X, E_{i}\right]_{\mathfrak{M}}\right\rangle E_{j}\right\}, E_{i}\right\rangle \\
\quad=\frac{1}{2} \sum_{i}\left\langle\left[\left[X, E_{i}\right]_{\mathfrak{M}}, X\right]_{\mathfrak{M}}, E_{i}\right\rangle-\frac{1}{2} \sum_{i}\left\|\left[X, E_{i}\right]_{\mathfrak{M}}\right\|^{2} .
\end{aligned}
$$

Using (3.7) now, the sum of the first two summands of the right-hand side of (3.5) is

$$
\begin{aligned}
-\sum_{i}\left\langle\alpha\left(X, \alpha\left(E_{i}, X\right)\right), E_{i}\right\rangle+\sum_{i}\left\langle\alpha\left(\left[X, E_{i}\right]_{\mathfrak{M}}, X\right), E_{\mathfrak{i}}\right\rangle \\
=+\frac{1}{4} \sum_{i}\left\langle\left[\left[X, E_{i}\right]_{\mathfrak{M}}, X\right]_{\mathfrak{M}}, E_{i}\right\rangle-\frac{1}{2} \sum_{i}\left\|\left[X, E_{i}\right]_{\mathfrak{M}}\right\|^{2} \\
-\quad-\frac{1}{4} \sum_{i, j}\left\langle\left[X, E_{i}\right]_{\mathfrak{M}}, E_{j}\right\rangle \cdot\left\langle\left[X, E_{j}\right]_{\mathfrak{M}}, E_{i}\right\rangle .
\end{aligned}
$$

Adding $\sum_{i}\left\langle\left[\left[X, E_{i}\right]_{\AA}, X\right]_{\Re \Re}, E_{i}\right\rangle$ to both sides of (3.8), our assertion (3.2) follows from (3.5). Q.E.D.

We apply Lemma 3.1 to the gap between the nilpotent and solvable radicals of $G$.

3.9. THEOREM. Let $\left(M, d s^{2}\right)$ be a riemannian homogeneous space, $G$ a transitive Lie group of isometries, $K$ the isotropy subgroup at a point $\mathcal{O} \in M$, and $\mathfrak{L}$ an $\operatorname{ad}_{G}(K)$ invariant Levi factor of $\mathbb{S}$, such that $d^{2}$ is consistent with the Levi-Whitehead decomposition $\mathbb{S}=\mathfrak{R}+\mathfrak{Q}$. Choose an invariant complement $\mathfrak{M}=\mathfrak{R}+\mathfrak{A}+(\mathfrak{M} \cap \mathfrak{L})$ 
for $K$ that satisfies (2.8). Let $X \in \mathfrak{M}$ be a unit vector, $X_{\mathcal{O}} \in M_{\mathcal{O}}$ the corresponding unit tangent vector.

1. If $X \perp[\mathfrak{S}, \mathbb{S}]_{\mathfrak{M}}$ then the mean curvature $\boldsymbol{k}\left(X_{\mathcal{O}}\right) \leqq 0$, and $\boldsymbol{k}\left(X_{\mathcal{O}}\right)=0$ if and only if (a) $X \in \mathfrak{N}$ and (b) $[X, \mathfrak{M}]=0$.

2. If $X \in \mathfrak{A}$ then $\boldsymbol{k}\left(X_{\mathcal{O}}\right)<0$.

Proof. By choice of $\mathfrak{M}$ and by [SS, $\mathbb{S}] \subset \mathfrak{R}+\mathfrak{Q}$ we have an orthogonal direct sum decomposition

$$
\begin{gathered}
\mathfrak{M}=\mathfrak{N}^{\prime}+\mathfrak{B}+(\mathfrak{M} \cap \mathfrak{L}), \quad \mathfrak{N}^{\prime}+\mathfrak{B}=\mathfrak{M} \cap \mathfrak{R}, \quad \mathfrak{N}^{\prime} \subset \mathfrak{R}, \\
{[\mathbb{S}, \mathbb{S}]_{\mathfrak{M}}=\mathfrak{N}^{\prime}+(\mathfrak{M} \cap \mathfrak{L}), \quad \mathfrak{U} \subset \mathfrak{B} .}
\end{gathered}
$$

Let $X \in \mathfrak{B}$. Then we have an orthonormal basis $\left\{E_{i}\right\}$ of $\mathfrak{M}$ containing $X$, such that each $E_{i}$ is in $\mathfrak{R}^{\prime}, \mathfrak{M} \cap \mathfrak{L}$ or $\mathfrak{B}$. We apply Lemma 3.1 with that basis.

Define coefficients by $\left[X, E_{j}\right]_{\Re}=\sum_{k} a_{j k} E_{k}$ and let $A=\left(a_{j k}\right)$. Then

$$
\left\langle\left[X, E_{i}\right]_{\mathfrak{M}}, E_{j}\right\rangle=a_{i j} \text { and }\left\langle\left[X, E_{j}\right]_{\mathfrak{R}}, E_{i}\right\rangle=a_{j i}
$$

so

$$
\sum_{i, j}\left\langle\left[X, E_{i}\right]_{\mathfrak{M}}, E_{j}\right\rangle\left\langle\left[X, E_{j}\right]_{\mathfrak{M}}, E_{i}\right\rangle=\operatorname{trace}\left(A^{\cdot t} A\right)
$$

Take polar decomposition $A=S T$ with $S$ symmetric and $T$ orthogonal. Then $A \cdot{ }^{t} A=S \cdot{ }^{t} S$. Let $S=\left(s_{i j}\right)$, so

$$
\operatorname{trace}\left(A \cdot{ }^{t} A\right)=\operatorname{trace}\left(S \cdot{ }^{t} S\right)=\sum_{i, j} s_{i j}^{2} \geqq 0,
$$

and note that $\sum s_{i j}^{2}=0$ if and only if $S=0$, which is equivalent to $A=0$. Thus

$$
-\frac{1}{4} \sum_{i, j}\left\langle\left[X, E_{i}\right]_{\mathfrak{M} i}, E_{j}\right\rangle\left\langle\left[X, E_{j}\right]_{\mathfrak{M}}, E_{i}\right\rangle \leqq 0
$$

with equality if and only if $[X, \mathfrak{M}]_{\mathfrak{M}}=0$.

That takes care of the last summand of (3.2). For the first two summands we define

$$
k_{i}=\left\langle\left[\left\{\left[X, E_{i}\right]_{\mathbb{R}}+\frac{1}{4}\left[X, E_{i}\right]_{\mathfrak{R}}\right\}, X\right]_{\mathfrak{R}}, E_{i}\right\rangle-\frac{1}{2}\left\|\left[X, E_{i}\right]_{\mathfrak{R}}\right\|^{2} .
$$

If $E_{i} \in \mathfrak{B}$ then $\left\langle[\mathfrak{S}, \mathfrak{S}]_{\mathfrak{M}}, \mathfrak{B}\right\rangle=0$ implies $k_{\mathfrak{i}}=-\frac{1}{2}\left\|\left[X, E_{\mathfrak{i}}\right]_{\mathfrak{M}}\right\|^{2}$. If $E_{\mathfrak{i}} \in \mathfrak{M} \cap \mathfrak{Q}$ then $[\mathscr{S}, X]_{\mathfrak{M}} \subset[\mathfrak{S}, \mathfrak{R}]_{\mathfrak{M}} \subset(\mathfrak{M} \cap \mathfrak{R}) \perp(\mathfrak{M} \cap \mathfrak{L})$ implies $\left\langle[\mathbb{S}, X]_{\mathfrak{M}}, E_{i}\right\rangle=0$ so

Thus

$$
k_{i}=-\frac{1}{2}\left\|\left[X, E_{i}\right]_{\mathfrak{M}}\right\|^{2} .
$$

$$
\text { if } E_{i} \in \mathfrak{B}+(\mathfrak{M} \cap \mathfrak{L}) \text { then } k_{i}=-\frac{1}{2}\left\|\left[X, E_{i}\right]_{\mathfrak{M}}\right\|^{2} \leqq 0 \text {. }
$$

If $E_{i} \in \mathfrak{R}^{\prime}$ then $E_{i} \in \mathfrak{N}$ so $\left[X, E_{i}\right] \in \mathfrak{R} \subset \mathfrak{M}$. Thus

$$
\text { if } E_{i} \in \mathfrak{N}^{\prime} \text { then } k_{i}=-\frac{1}{4}\left\langle\operatorname{ad}(X)^{2} E_{i}, E_{i}\right\rangle-\frac{1}{2}\left\|\left[X, E_{i}\right]\right\|^{2} \text {. }
$$


As $($ ad $X) \mathfrak{N}^{\prime} \subset \mathfrak{N}^{\prime}$ we can stipulate that, for numbers $\left\{\lambda_{b}\right\}$ such that $\left\{\lambda_{b}, \lambda_{b}\right\}$ are the eigenvalues of $\left.(\operatorname{ad} X)\right|_{\mathfrak{R}}$, each $E_{\mathfrak{i}} \in \mathfrak{N}^{\prime}$ is contained in the sum of the subspaces of $\mathfrak{N}^{\prime C}$ on which (for some $b=b_{i}$ ) ad $X-\lambda_{b}$ and ad $X-\lambda_{b}$ are nilpotent. That stipulation made, $\left\|\left[X, E_{i}\right]\right\|^{2} \geqq\left|\lambda_{b}\right|^{2}$ and $\left|\left\langle\operatorname{ad}(X)^{2} E_{i}, E_{i}\right\rangle\right| \leqq\left|\lambda_{b}\right|^{2}$. So (3.14) implies

$$
\text { if } E_{i} \in \mathfrak{N}^{\prime} \text { then } k_{i} \leqq-\frac{1}{4}\left|\lambda_{b}\right|^{2} \leqq 0 .
$$

Combining (3.13) and (3.15) we have $\sum_{i} k_{i} \leqq 0$. Adding that inequality to (3.11), and applying Lemma 3.1, we conclude

$$
\boldsymbol{k}\left(X_{\mathcal{O}}\right) \leqq 0 \text { with equality if and only if }[X, \mathfrak{M}]_{\mathfrak{M}}=0 .
$$

If $[X, \mathfrak{M}]_{\mathfrak{R}}=0$ then $[X, \mathfrak{M}] \subset \mathfrak{\Re}$. As $\mathfrak{R} \subset \mathfrak{M}$ and $[X, \mathfrak{R}] \subset \mathfrak{R}$ it follows that $[X, \mathfrak{R}]$ $=0$. Then $\mathfrak{S}=X R+\mathfrak{R}$ is a nilpotent subalgebra of $\mathfrak{S}$. But $X \in \mathfrak{R}$ and $[\mathfrak{R}, \mathfrak{R}] \subset \mathfrak{N}$, so $\mathfrak{S}$ is a nilpotent ideal in $\Re$. As $\mathfrak{R}$ is the maximal nilpotent ideal of $\Re$ it follows that $X \in \mathfrak{R}$. This fact and (3.16) imply the first statement of Theorem 3.9. If $X \in \mathfrak{A}$ then $X \notin \Re$, so $\boldsymbol{k}\left(X_{\mathcal{O}}\right)<0$. That completes the proof of Theorem 3.9. Q.E.D.

4. Application to manifolds of nonnegative mean curvature. We first apply Theorem 3.9 to homogeneous riemannian manifolds.

4.1. TheOREM. Let $\left(M, d s^{2}\right)$ be a connected homogeneous riemannian manifold, $G$ a transitive Lie group of isometries, $K$ an isotropy subgroup, and $\mathfrak{L}$ an $\operatorname{ad}_{G}(K)$ invariant Levi factor of (S) such that $d s^{2}$ is consistent with the Levi-Whitehead decomposition $\mathbb{B}=\mathfrak{R}+\mathfrak{R}$.

1. If $\left(M, d s^{2}\right)$ has mean curvature $\geqq 0$ everywhere, then the solvable radical $\Re$ and the nilpotent radical $\mathfrak{R}$ of (S) satisfy $\mathfrak{R}=\mathfrak{R}+(\mathfrak{R} \cap \mathfrak{R})$ semidirect sum.

2. If $\left(M, d s^{2}\right)$ has mean curvature $>0$ everywhere, then the derived group $[G, G]$ of $G$ is transitive on $M$.

Proof. If $\left(M, d s^{2}\right)$ has mean curvature $\geqq 0$ everywhere, then, in the notation (2.8), Theorem 3.9 says $\mathfrak{A}=0$, so $\mathfrak{M} \cap \mathfrak{R}=\mathfrak{R}$; thus $\mathfrak{R}=(\mathfrak{M} \cap \mathfrak{R})+(\mathfrak{R} \cap \mathfrak{R})$ $=\mathfrak{R}+(\Re \cap \Re)$. If further $\left(M, d s^{2}\right)$ has mean curvature $>0$ everywhere, then Theorem 3.9 says $\mathfrak{M}=[\mathscr{S}, \mathcal{S}]_{\mathfrak{M}}$, so the derived group $G^{\prime}=[G, G]$ has an open orbit $G^{\prime}(\mathcal{O}) \subset M$. As $G^{\prime}(\mathcal{O})$ is complete and $M$ is connected, $G^{\prime}(\mathcal{O})=M$, so $G^{\prime}$ is transitive on $M$. Q.E.D.

4.2. COROLlary. Let $\left(M, d s^{2}\right)$ be a connected riemannian homogeneous manifold, $G$ a transitive Lie group of isometries of $M, \mathcal{O} \in M$, and $K$ the isotropy subgroup of $G$ at $\mathcal{O}$. Let $R_{\mathcal{O}}$ denote the subspace of the tangent space $M_{\mathcal{O}}$ consisting of vectors $Y_{\mathcal{O}}$ where $Y$ is contained in the solvable radical $\Re$ of $\mathscr{S}$. Suppose that the linear isotropy representation of $K$ splits into disjoint representations on $R_{\mathcal{O}}$ and $R_{\mathcal{O}}^{\perp}$.

1. If $\left(M, d s^{2}\right)$ has mean curvature $\geqq 0$ everywhere, then $\Re$ is related to the nilpotent radical $\mathfrak{R}$ of $\mathfrak{S}$ by $\mathfrak{R}=\mathfrak{R}+(\mathfrak{\Re} \cap \mathfrak{R})$. 
2. If $\left(M, d s^{2}\right)$ has mean curvature $>0$ everywhere, then the derived group of $G$ is transitive on $M$.

Proof. Let $\mathfrak{L}$ be any $\operatorname{ad}_{G}(K)$-invariant Levi factor of $\mathbb{S}$. Proposition 2.4 says that $d s^{2}$ is consistent with the Levi-Whitehead decomposition $\mathscr{S}=\mathfrak{R}+\mathfrak{Q}$. Our assertions now follow from Theorem 4.1. Q.E.D.

In order to apply Theorem 4.1 to the case of a transitive solvable group of isometries, we must first prove the following lemma about a simply transitive nilpotent group of isometries. Note that the lemma extends the positive curvature portion of [7].

4.3. Lemma. Let $\left(N, d s^{2}\right)$ be a connected nilpotent Lie group with a left invariant riemannian metric. Then the following conditions are equivalent.

(i) $\left(N, d s^{2}\right)$ has mean curvature $\geqq 0$ everywhere.

(ii) $\left(N, d s^{2}\right)$ has every sectional curvature zero.

(iii) $N$ is commutative.

Proof. As (iii) $\Rightarrow$ (ii) $\Rightarrow$ (i) trivially we need only check that (i) $\Rightarrow$ (iii). So assume that $\left(N, d s^{2}\right)$ has mean curvature $\geqq 0$ everywhere. In the context of Theorem 3.9 ,

$$
G=N, \quad K=\{1\}, \quad \mathfrak{Q}=0, \quad \mathfrak{M}=\mathfrak{R},
$$

and consistency of $d s^{2}$ with $\mathbb{S}=\Re+\mathfrak{Q}$ is automatic. Now Theorem 3.9 says that there is no noncentral element $X \in \mathfrak{N}$ such that $X \perp[\mathfrak{N}, \mathfrak{N}]$. But nilpotence of $\mathfrak{N}$ implies that in the lower central series

$$
\mathfrak{N}=\mathfrak{R}_{0} \supset \mathfrak{N}_{1} \supset \ldots \supset N_{s} \supsetneqq \mathfrak{N}_{s+1}=0, \quad \mathfrak{R}_{n+1}=\left[\mathfrak{R}, \mathfrak{N}_{k}\right],
$$

any vector space complement to $\mathfrak{R}_{1}=[\mathfrak{R}, \mathfrak{R}]$ generates $\mathfrak{R}$. Let $[\mathfrak{R}, \mathfrak{R}]^{\perp}$ be the complement. As it consists of central elements of $\mathfrak{R}$ (our application of Theorem 3.9), it must be all of $\mathfrak{R}$. Thus $N$ is commutative. Q.E.D.

Now we have a general result on the curvature of riemannian solvmanifolds.

4.4. THEOREM. Let $\left(M, d s^{2}\right)$ be a connected riemannian manifold that has a solvable transitive group of isometries. Then the following conditions are equivalent.

(i) $\left(M, d s^{2}\right)$ has mean curvature $\geqq 0$ everywhere.

(ii) $\left(M, d s^{2}\right)$ has every sectional curvature $\geqq 0$.

(iii) $\left(M, d s^{2}\right)$ has every sectional curvature zero.

(iv) $\left(M, d s^{2}\right)$ is isometric to the product of an euclidean space and a flat riemannian torus.

Proof. As (iv) $\Rightarrow$ (iii) $\Rightarrow$ (ii) $\Rightarrow$ (i) trivially we need only check that (i) $\Rightarrow$ (iv). So assume that $\left(M, d s^{2}\right)$ has mean curvature $\geqq 0$. 
$G$ denotes the closure of a solvable transitive group of isometries of $\left(M, d s^{2}\right)$ in the full group of isometries. So $G$ is a solvable transitive Lie group of isometries. Let $K$ be an isotropy subgroup. (S) is its own solvable radical $\Re$, so the $\operatorname{ad}_{G}(K)$ invariant Levi factor $\mathfrak{L}=0$, and $d s^{2}$ is consistent with $\mathfrak{S}=\mathfrak{R}+\mathfrak{L}=\mathfrak{R}$. Our invariant complement $\mathfrak{M}$ for $K$ satisfying (2.8) now has the form $\mathfrak{M}=\mathfrak{R}+\mathfrak{A}$, and Theorem 3.9 says $\mathfrak{A}=0$.

Let $N$ be the analytic subgroup of $G$ for $\Re$. Now we have an open orbit $N(\mathcal{O}) \subset M$. As $N(\mathcal{O})$ is complete and $M$ is connected, the two are equal. Thus $N$ is transitive on $M$. As $G$ acts effectively on $M$, also $N$ acts effectively, so Lemma 2.5 says $K \cap N$ $=\{1\}$. That proves $N$ simply transitive on $M$. Lemma 4.3 says that $\left(M, d s^{2}\right)$ is flat and $N$ is commutative. It follows [6, Théorème 4] that $\left(M, d s^{2}\right)$ is the product of an euclidean space and a flat riemannian torus. Q.E.D.

4.5. COROllary. Let $\left(M, d s^{2}\right)$ be a connected riemannian Einstein manifold that has a solvable transitive group of isometries. Then either $\left(M, d s^{2}\right)$ has vanishing Ricci tensor and is isometric to the product of an euclidean space with a flat riemannian torus, or $\left(M, d s^{2}\right)$ has negative definite Ricci tensor.

Proof. The Einstein homogeneous hypothesis says that $\left(M, d s^{2}\right)$ has constant mean curvature, say $k$. If $k \geqq 0$ then Theorem 4.4 says that $\left(R_{i j}\right) \equiv 0$ and that $\left(M, d s^{2}\right)$ is the product of an euclidean space with a flat riemannian torus. If $k<0$ then $\left(R_{i j}\right)$ is negative definite. Q.E.D.

For examples of the latter case of Corollary 4.5 , let $\left(M, d s^{2}\right)$ be a noncompact irreducible riemannian symmetric space, $G$ the largest connected group of isometries, $K$ an isotropy subgroup, and $G=N A K$ an Iwasawa decomposition. Then $S=N A$ is a simply transitive solvable Lie group of isometries of $\left(M, d s^{2}\right)$, and $\left(M, d s^{2}\right)$ is a connected riemannian Einstein manifold with negative definite Ricci tensor. G. Jensen [2] has shown that this example is essentially exhaustive in dimensions $\leqq 4$.

The following lemma is similar to results of G. Jensen [2].

4.6. Lemma. Let $G$ be a Lie group, let $d s^{2}$ be a left invariant riemannian metric on $G$, and let $X$ be a nonzero central element of the Lie algebra $\mathbb{S}$. Then the mean curvature $\boldsymbol{k}(X) \geqq 0$, and $\boldsymbol{k}(X)=0$ if and only if $X$ is orthogonal to the derived algebra of (S).

Proof. We use the notation of the proof of Lemma 3.1. Note $\mathfrak{M}=\mathscr{S}$. We take $X$ to be a unit vector and $\left\{E_{i}\right\}$ to be an orthonormal basis of \&s that contains $X$. Then (3.3) holds. As $X$ is central in S, the analog of (3.4) is

$$
\mathscr{U}(S, X)=\mathscr{U}(X, S)=-\frac{1}{2} \sum_{j}\left\langle\left[S, E_{j}\right], X\right\rangle E_{j}
$$


We still have $\mathscr{U}(X, X)=0$, so $\alpha(X, X)=0$ and (3.5) holds. But $\left[X, E_{i}\right]=0$ simplifies (3.5) to

$$
\begin{aligned}
(n-1) \boldsymbol{k}(X) & =-\sum_{i}\left\langle\alpha\left(X, \alpha\left(E_{i}, X\right)\right), E_{i}\right\rangle \\
& =\frac{1}{2} \sum_{i, j}\left\langle\alpha\left(X,\left\langle\left[E_{i}, E_{j}\right], X\right\rangle E_{j}\right), E_{i}\right\rangle \\
& =\frac{1}{2} \sum_{i, j}\left\langle\left[E_{i}, E_{j}\right], X\right\rangle \cdot\left\langle\alpha\left(X, E_{j}\right), E_{i}\right\rangle \\
& =-\frac{1}{4} \sum_{i, j, k}\left\langle\left[E_{i}, E_{j}\right], X\right\rangle\left\langle\left\langle\left[E_{j}, E_{k}\right], X\right\rangle E_{k}, E_{i}\right\rangle \\
& =-\frac{1}{4} \sum_{i, j}\left\langle\left[E_{i}, E_{j}\right], X\right\rangle\left\langle\left[E_{j}, E_{i}\right], X\right\rangle \\
& =\frac{1}{4} \sum_{i, j}\left\langle\left[E_{i}, E_{j}\right], X\right\rangle^{2} .
\end{aligned}
$$

Thus $\boldsymbol{k}(X) \geqq 0$, and $\boldsymbol{k}(X)=0$ if and only if each $\left\langle\left[E_{i}, E_{j}\right], X\right\rangle=0$, which is equivalent to $\langle[$ SS, (S) $], X\rangle=0$. Q.E.D.

We now combine Lemmas 4.3 and 4.6, extending our calculations [7] from sectional curvature to mean curvature, and sharpening Theorem 4.4 in the case of a nilpotent group. After hearing the result, G. Jensen gave another proof of Theorem 4.7 [2, Theorem 4].

4.7. THEOREM. Let $\left(M, d s^{2}\right)$ be a connected riemannian manifold that has a nilpotent transitive group of isometries. Then the following conditions are equivalent.

(i) $\left(M, d s^{2}\right)$ has mean curvature $\geqq 0$ everywhere.

(ii) $\left(M, d s^{2}\right)$ has mean curvature $=0$ everywhere.

(iii) $\left(M, d s^{2}\right)$ has mean curvature $\leqq 0$ everywhere.

(iv) $\left(M, d s^{2}\right)$ has every sectional curvature $\geqq 0$.

(v) $\left(M, d s^{2}\right)$ has every sectional curvature $=0$.

(vi) $\left(M, d s^{2}\right)$ has every sectional curvature $\leqq 0$.

(vii) $\left(M, d s^{2}\right)$ is isometric to the product of an euclidean space and a flat riemannian torus.

Proof. Let $N$ denote the identity component of the closure of a nilpotent transitive group of isometries. Then $N$ is a connected nilpotent transitive Lie group of isometries of $\left(M, d s^{2}\right)$. Its isotropy subgroups are central by Lemma 2.5 , hence trivial; thus $N$ is simply transitive on $\left(M, d s^{2}\right)$. Now we may view $d s^{2}$ as a left invariant riemannian metric on $N$.

Lemma 4.3 says that (i) implies (v); so (ii) implies (v).

We use Lemma 4.6 to prove that (iii) implies (v). Let 8 be the last nonzero term of the lower central series of $\mathfrak{R}$. Then 3 is central in $\mathfrak{R}$. Let $0 \neq X \in \mathbb{3}$. Assume (iii), so $\boldsymbol{k}(X) \leqq 0$. Lemma 4.6 says $\boldsymbol{k}(X) \geqq 0$. Thus $\boldsymbol{k}(X)=0$ and Lemma 4.6 says $\langle[\mathfrak{N}, \mathfrak{R}], X\rangle=0$. If $\mathfrak{N}$ is noncommutative then $X \in \mathfrak{Z} \subset[\mathfrak{N}, \mathfrak{N}]$ and so $\langle[\mathfrak{N}, \mathfrak{R}], X\rangle$ 
$\neq 0$. That proves $N$ commutative, so every sectional curvature of $\left(N, d s^{2}\right)$ is zero. Thus (iii) implies (v).

Now (i), (ii) and (iii) each implies (v). It follows that (iv) and (vi) each implies (v). But (v) implies (vii) by [6, Théorème 4], and (vii) clearly implies each of (i), (ii), (iii), (iv), (v) and (vi). Q.E.D.

4.8. CoRollary. Let $\left(M, d s^{2}\right)$ be a connected riemannian Einstein manifold that has a nilpotent transitive group of isometries. Then $\left(M, d s^{2}\right)$ is isometric to the product of an euclidean space and a flat riemannian torus.

Proof. We have the hypothesis of Theorem 4.7 as well as condition (i), (ii) or (iii); thus we have condition (vii) of Theorem 4.7. Q.E.D.

Our last application is a refinement of [8, Corollary 5.8].

4.9. THEOREM. Let $\left(M, d s^{2}\right)$ be a compact connected locally homogeneous riemannian manifold with mean curvature $\geqq 0$ everywhere. Suppose that the fundamental group $\pi_{1}(M)$ has a solvable subgroup of finite index.

Let $\pi: \tilde{M} \rightarrow M$ be the universal riemannian covering, $G$ the largest connected group of isometries of $\left(\tilde{M}, \pi^{*} d s^{2}\right), K$ an isotropy subgroup of $G, \mathfrak{I}$ an $\operatorname{ad}_{G}(K)-$ invariant Levi factor of $\mathscr{S}$ and $L$ its analytic subgroup of $G$, and $\Re$ and $\Re$ the solvable and nilpotent radicals of $S S$ and $R$ and $N$ their analytic subgroups of $G$. Let $\Gamma$ denote the group $\cong \pi_{1}(M)$ of deck transformations of $\tilde{M} \rightarrow M$. Suppose that $\pi^{*} d s^{2}$ is consistent with $\mathfrak{S H}=\Re+\mathfrak{R}$.

\section{L is compact.}

2. $R=N \cdot(K \cap R)_{0}$ semidirect product, where $(K \cap R)_{0}$ is a torus group whose Lie algebra $\mathfrak{R} \cap \Re$ acts effectively on $\mathfrak{R}$ in the adjoint representation.

3. $\Gamma$ has a torsion free normal nilpotent subgroup $\Delta$ of finite index, $\Delta \subset N \cdot Z_{L}(N)_{0}$ where $Z_{L}(N)$ is the centralizer of $N$ in $L$, and $\Delta$ projects isomorphically to a discrete subgroup with compact quotient in $N$.

Proof. Compactness of $L$ is part of [8, Corollary 5.8], and the decomposition $R=N \cdot(K \cap R)_{0}$ follows from Theorem 4.1. In the proof of [8, Corollary 5.8] it is shown that $\Gamma$ has a nilpotent subgroup $\Delta$ of finite index, and that the identity component of the closure of $R \Delta$ in $G$ has form

$$
F=R \cdot U \text { for some torus } U \subset L \text {. }
$$

Enlarge $U$ to a maximal torus $T$ of $F$. Then $T=T_{N} \cdot T_{R / N} \cdot U$ local direct product, where $T_{N}$ is a maximal torus of $N$ and $T_{R / N}$ is an $\operatorname{ad}_{G}(R)$-conjugate of $(K \cap R)_{0}$. These constructions are not changed if $\Delta$ is cut down to a subgroup of finite index. So we first cut $\Delta$ down to $\Delta \cap F$, then [8, (4.5)] to a torsion free group, and finally to a normal subgroup of $\Gamma$.

Let $V$ be the kernel of the action of $T$ on $N$, i.e. the centralizer $Z_{T}(N)$. Then $T_{N} \subset V_{0} \subset T_{N} \cdot U$. Let $F^{*}=F / V$; then $F^{*}=N^{*} \cdot T^{*}$ where $N^{*}=N / T_{N}$ simply connected nilpotent group and

$$
T^{*}=\left(T_{R / N} \cdot U\right) /\left\{\left(T_{R / N} \cdot U\right) \cap V\right\} .
$$


$\Delta^{*}$ is the projection of $\Delta$ to $F^{*}$. As $\Delta$ is a discrete subgroup with compact quotient in $F$, the same is true of $\Delta^{*}$ in $F^{*}$. By construction of $F^{*}$ and the fact that $T_{N}$ is central in $F$, conjugation represents $T^{*}$ faithfully as a group of automorphisms of $N^{*}$, so L. Auslander's result [1] says that $\Delta^{*} \cap N^{*}$ has finite index in $\Delta^{*}$. Again cutting $\Delta$ down, we may assume $\Delta^{*} \subset N^{*}$, i.e. that $\Delta \subset N \cdot Z_{U}(N)_{0} \subset N \cdot Z_{L}(N)_{0}$. Q.E.D.

\section{REFERENCES}

1. L. Auslander, Bieberbach's theorems on space groups and discrete uniform subgroups of Lie groups, Ann. of Math. 71 (1960), 579-590.

2. G. Jensen, Homogeneous Einstein spaces, Thesis, University of California, Berkeley, 1968.

3. G. D. Mostow, Fully reducible subgroups of algebraic groups, Amer. J. Math. 78 (1956), 200-221.

4. K. Nomizu, Invariant affine connections on homogeneous spaces, Amer. J. Math. 76 (1954), 33-65.

5. E. J. Taft, Orthogonal conjugacies in associative and Lie algebras, Trans. Amer. Math. Soc. 113 (1964), 18-29.

6. J. A. Wolf, Sur la classification des variétés riemanniennes homogènes à courbure constante, C. R. Acad. Sci. Paris 250 (1960), 3443-3445.

7. — Curvature in nilpotent Lie groups, Proc. Amer. Math. Soc. 15 (1964), 271-274.

8. - Growth of finitely generated solvable groups and curvature of riemannian manifolds, J. Differential Geometry, (to appear).

UNIVERSITY OF CALIFORNIA,

Berkeley, California 